\title{
Uma Análise do Uso da Robótica Educacional como Ferramenta de Aprendizagem em Lógica de Programação
}

\author{
Eduardo Cambruzzi $^{1 *}$, Jessica de S. Santana ${ }^{1}$ \\ ${ }^{1}$ Instituto Federal de Educação, Ciência e Tecnologia da Bahia - IFBA \\ Valença - Bahia - Brasil \\ ec@ifba.edu.br, jhessiica.santana@gmail.com
}

\begin{abstract}
In the last decades the interest about the Educational Robotics as a teaching-learning tool in disciplines related to computer programming. However, there are few studies that evaluate its use on a long term, whether in learning or about students interest in the area of computing. In this article we present the quantitative and qualitative results of the use of robotics as a teaching-learning tool in Programming Logic over the three years in high school student.
\end{abstract}

Resumo. Nas últimas décadas, tem crescido o interesse pelo uso da Robótica Educativa como ferramenta de ensino-aprendizagem em disciplinas relacionadas à programação de computadores. No entanto, poucos são os estudos que avaliam os impactos de longo prazo de seu uso, seja na aprendizagem ou no interesse dos alunos pela área da computação. Neste artigo, apresentam-se os resultados quantitativos e qualitativos do uso da robótica como ferramenta de ensino-aprendizagem de Lógica de Programação ao longo dos três anos de formação de alunos do ensino médio-técnico.

\section{Introdução}

A dificuldade em abstrair problemas e transformá-los em comandos para serem executados por um computador, estão entre os grandes desafios que permeiam o desenvolvimento do raciocínio lógico computacional. Para [Piva Jr and Freitas 2011], alunos que não desenvolvem esta competência logo no inıcio dos cursos de computação, tendem a se desmotivar e abandonar estes cursos. Assim, buscando melhorar o ensino e a aprendizagem de disciplinas que exigem o desenvolvimento do raciocínio lógico computacional, a Robótica Educacional (RE) tem sido utilizada como uma ferramenta, através da qual os alunos podem compreender e aplicar na prática conceitos abstratos e complexos [Melchior et al. 2005]. É por este motivo que cada vez mais a RE tem sido agregada ao currículo dos cursos de engenharia e computação, como forma de tornar o aprendizado mais atrativo e menos abstrato. Vários estudos como os apresentados por [Gorakhnath and Padmanabhan 2017], [Piva Jr and Freitas 2011], [Gorakhnath and Padmanabhan 2017], demonstram que grupos de alunos que utilizam a RE aumentam seu interesse e melhoram seu aproveitamento em Lógica de Programação. No entanto, estes estudos não avaliam o impacto de longo prazo do uso da RE na aprendizagem de lógica de programação e no interesse dos alunos em ingressar ou se manter na área de computação. Neste artigo, apresentam-se os resultados de um estudo com duração

*Agradecemos ao Instituto Federal de Educação, Ciência e Tecnologia da Bahia - IFBA 
de três anos, no qual avalia-se se o uso da Robótica Educacional contribui de forma duradoura na aprendizagem, no interesse e no desempenho dos alunos nas disciplinas de programação de computadores no curso técnico de informática do Instituto Federal de Educação, Ciência e Tecnologia da Bahia, campus Valença.

\subsection{Robótica Educacional como ferramenta de ensino}

A principal forma de aplicação da RE em sala de aula, deriva das teorias Construcionistas de [Papert 1986]. Segundo Papert, a resolução de problemas reais incentiva a criação de soluções complexas através da tecnologia utilizada, envolvendo e motivando os alunos a aprenderem à medida que se pensa e age sobre o objeto. É este ambiente lúdico, interativo e participativo, propiciado pela RE, que permite ao aluno compreender e resolver problemas abstratos e complexos, que envolvam raciocínio lógico e abstração, através da construção e manipulação de soluções concretas na forma de robôs. Graças à flexibilidade da RE, esta pode ser utilizada em deferentes áreas do conhecimento. Isto permite que educadores explorem conceitos abstratos de difícil compreensão de forma concreta e lúdica. A RE proporciona um ambiente de aprendizagem no qual os alunos se envolvem no projeto, na construção e na programação de robôs. Além disso, inclui atividades que fortalecem o trabalho em equipe, a construção do conhecimento e a inovação, sempre com o intuito de resolver problemas práticos [Gorakhnath and Padmanabhan 2017]. Tais afirmações são baseadas em resultados de vários estudos, como os realizados por [Piva Jr and Freitas 2011], [Paula et al. 2009], [Gorakhnath and Padmanabhan 2017]. Geralmente, estes estudos são aplicados a pequenos grupos de alunos, em atividades concomitantes à sua formação e em curtos espaços de tempo escolar dos alunos. Tais estudos não buscam identificar se o uso da RE apresentam impacto de longo prazo na aprendizagem de lógica de programação e no interesse dos alunos em ingressar ou se manter na área de computação.

\section{Ensino de Lógica de Programação}

As disciplinas de Lógica de Programação ou Algoritmos estão presente em todos os currículos de cursos técnicos ou superiores da área de Computação e historicamente apresentam um elevado índice de reprovação e desistência [Sirotheau et al. 2012]. Um dos fatores que dificultam o aprendizado destas disciplinas é a didática adotada em sala de aula. Segundo [Jenkins 2002], as metodologias tradicionalmente utilizadas não conseguem motivar os alunos, de modo que estes perdem o interesse pela disciplina. Já de acordo com [Paula et al. 2009], há uma dificuldade para que os alunos consigam construir abstrações mentais a partir de problemas concretos, sendo necessário buscar meios que estimulem os alunos a desenvolverem tal capacidade. É neste contexto de dificuldade metodológica e baixo interesse dos alunos pelas disciplinas de programação, que muitos autores têm apontado que o uso de metodologias de ensino mais lúdicas podem se tornar ferramentas úteis para atrair a atenção, o interesse e motivar alunos a aprenderem a programar. Segundo [Macedo et al. 2005], o uso de tais metodologias pode auxiliar no processo de aprendizagem, pois possuem as seguintes qualidades: 1) tornam as tarefas prazerosas; 2) são desafiadoras; 3) possuem dimensão simbólica; 4) não limitam as possibilidades e; 5) expressam-se de modo construtivo e relacional. Tais características resumem o contexto de aprendizagem propiciado pela RE e sua viabilidade de uso na relação ensino-aprendizagem nos cursos de computação. 


\section{Metodologia Utilizada}

Para obter os dados desta pesquisa, realizou-se um estudo de três anos com 58 alunos de duas turmas do curso médio integrado de informática do Instituto Federal de Educação, Ciência e Tecnologia da Bahia Campus Valença. Estas turmas, tratadas doravante por turma A (28 alunos) e turma B (30 alunos), possuem alunos com características sociais e etárias semelhantes e ambas utilizaram os mesmos recursos de hardware e software em laboratórios de informática da instituição.

A turma A utilizou a Robótica Educacional sob uma perspectiva Construcionista somente na disciplina de Lógica de Programação durante o primeiro ano do estudo. $\mathrm{O}$ objetivo desta escolha foi avaliar se o aprendizado através de uma metodologia lúdica, interativa e construcionista baseada em Robótica Educacional, contribui de maneira duradoura para o interesse e desempenho dos alunos, mesmo que estes sejam submetidos posteriormente ao ensino tradicional de programação. Já os alunos da turma B, que servem como turma de controle neste estudo, foram submetidos à metodologia de ensino Tradicional durante os três anos do estudo. Assume-se neste artigo, que a metodologia Tradicional baseia-se na exposição verbal por parte do professor e na preparação do aluno com foco na resolução de exercícios e na memorização de fórmulas e conceitos. Nesta, o professor realiza a preparação do aluno, e em seguida apresenta o conteúdo relacionandoo com outros assuntos e, por fim, é feita a generalização e aplicação de exercícios. Já na metodologia Construcionista proposta por Papert, a teoria e a aprendizagem ocorrem ao mesmo tempo. Nesta estratégia de ensino, cada sujeito é responsável por sua aprendizagem à medida que experimenta e constrói algo [Papert 1986]. Assim, os alunos são os protagonistas na ação de aprendizagem, envolvendo-se na definição das tarefas e na resolução de problemas. Ao final de cada ano letivo foram realizadas entrevistas com os alunos das turmas A e B, nas quais foram levantadas informações qualitativas sobre suas expectativas e percepções em relação às disciplinas de programação, ao curso e interesses profissionais. Também foram levantados dados quantitativos relacionados às notas destes alunos nas disciplinas de programação em cada ano letivo. A fim de manter a coerência dos resultados, as notas médias dos alunos foram normalizadas, uma vez que, isto permite obter valores mais precisos entre diferentes médias de disciplinas e alunos [Lindahl and Ramón 2010].

Algumas destas análises e percepções estão descritas na Seção 4.

\section{Análise dos Resultados}

Observando-se a Figura 1(a), e a Figura 1(b), pode-se afirmar que o uso da Robótica Educacional aplicada no primeiro ano do estudo na turma A, faz com que os alunos apresentem melhores resultados na compreensão e solução dos problemas. Nas mesmas figuras, observa-se que, no segundo ano, quando os alunos da turma A são submetidos a metodologia Tradicional, estes apresentam uma queda significativa na capacidade de compressão e resolução de problemas, entretanto, seus resultados em todos os anos do estudo são superiores aos da turma B. Isto pode ser um indicativo de que o uso da Robótica Educacional influência positivamente ao longo do curso, mesmo quando esta metodologia não é mais utilizada. Outro aspecto que deve ser observado nos resultados é o do interesse dos alunos pelas tarefas propostas em sala de aula, mostrado na Figura 1(d). Note que o percentual de alunos da turma B interessados pelas tarefas de programação, quase não 
apresenta diferenças ao longo dos anos. Porém, os alunos da turma A apresentam grande interesse por elas no primeiro ano, interesse que degrada rapidamente com a adoção da metodologia Tradicional, mas que se mantém superior aos da turma B em todos os anos do estudo. O que pode ser mais um indicativo da resiliência do uso da Robótica Educacional no primeiro ano na turma $\mathrm{A}$.

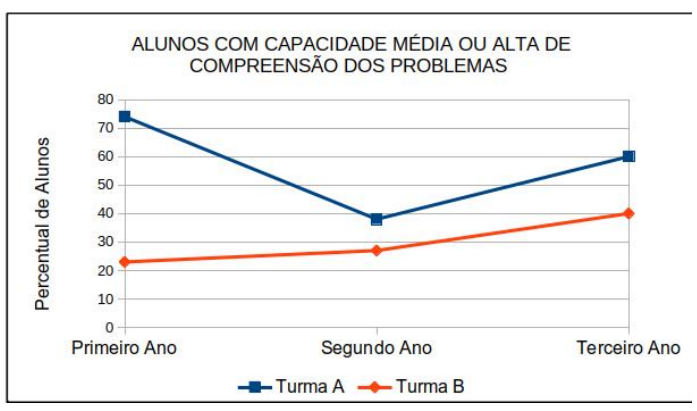

(a) Compreensão dos problemas abordados.

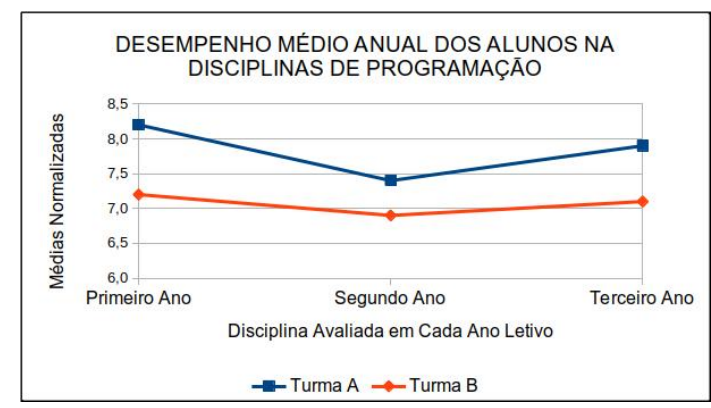

(c) Desempenho dos alunos nas disciplinas de programação.

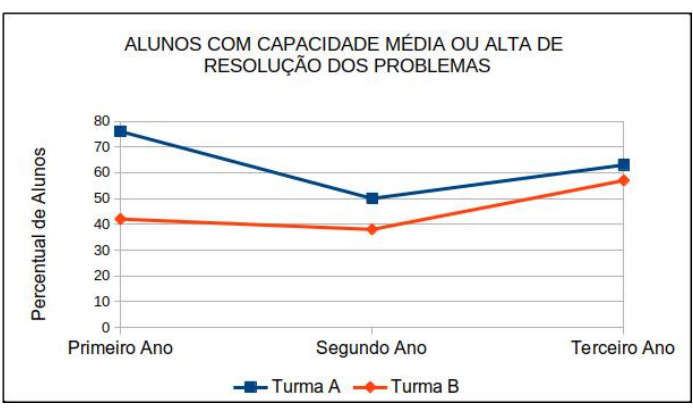

(b) Capacidade de resolução dos problemas abordados.

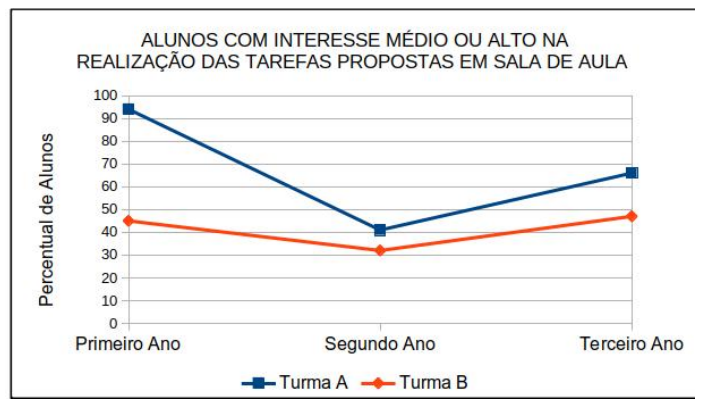

(d) Interesse pelas disciplinas de programação.

Figura 1. Relação entre os resultados qualitativos e quantitativos da pesquisa.

No entanto, ao comparar os resultados de desempenho quantitativo dos alunos, Figura 1(c), percebe-se que a diferença não é tão significativa ao longo dos anos. Porém, os alunos da turma B, possuem maiores dificuldades de compreender e solucionar problemas que os da turma A, como indicam os dados nas Figuras 1(a) e 1(b). Uma análise mais detalhada das respostas obtidas dos alunos da turma $\mathrm{B}$, indicou que estes memorizaram tarefas e comandos, sendo capazes de implementar códigos corretos, mas sem compreender os problemas associados à solução, uma vez que as avaliações repetiam problemas descritos e resolvidos em sala de aula. Ainda assim, é preciso salientar que, apesar dos resultados apontarem um impacto positivo da RE, tanto na aprendizagem de programação, como no posterior interesse dos alunos por computação, estes resultados podem ser afetados pelas escolhas metodológicas dos professores das demais disciplinas de programação cursadas ao longo dos três anos do curso. 


\section{Considerações Finais}

Este artigo apresenta alguns dos resultados obtidos em um estudo de longo prazo sobre o ensino de programação de computadores para alunos do ensino médio integrado do IFBA Campus Valença. O objetivo do estudo é observar se a utilização de metodologias baseadas na Robótica Educacional pode contribuir positivamente na relação ensinoaprendizagem destas disciplinas. Os resultados obtidos indicam que a Robótica Educacional colabora para um aprendizado mais significativo nas disciplinas de programação e que o uso desta metodologia contribui tanto na melhoria do desempenho dos alunos em todo ciclo de formação, como também aumenta o interesse pelos conteúdos abordados. Além disso, indica que seu uso em disciplinas iniciais nos cursos de computação, se reflete no desempenho e interesse dos alunos nos anos posteriores à sua utilização. Em estudos futuros, serão analisados dados relacionados ao uso da Robótica Educacional e o engajamento dos alunos no curso de computação e seu interesse em prosseguir profissionalmente nesta área.

\section{Referências}

Gorakhnath, I. and Padmanabhan, J. (2017). Educational robotics: A new arena in classroom teaching.

Jenkins, T. (2002). On the difficulty of learning to program. In Proceedings of the 3rd Annual Conference of the LTSN Centre for Information and Computer Sciences, volume 4, pages 53-58. Citeseer.

Lindahl, J. M. M. and Ramón, M. C. (2010). The generalized hybrid averaging operator and its application in decision making. Revista de Métodos Cuantitativos para la Economía y la Empresa, 9:69-84.

Macedo, L., Petty, A. L. S., and PASSOS, N. C. (2005). Os jogos e o lúdico na aprendizagem escolar.

Melchior, A., Cohen, F., Cutter, T., Leavitt, T., and Manchester, N. (2005). More than robots: An evaluation of the first robotics competition participant and institutional impacts. Heller School for Social Policy and Management, Brandeis University.

Papert, S. (1986). Constructionism: A new opportunity for elementary science education. Massachusetts Institute of Technology, Media Laboratory, Epistemology and ....

Paula, L. Q., Júnior, D. P., and Freitas, R. L. (2009). A leitura e a abstração do problema no processo de formação do raciocínio lógico-abstrato em alunos de computação. ReverteRevista de Estudos e Reflexões Tecnológicas da Faculdade de Indaiatuba, (7).

Piva Jr, D. and Freitas, R. L. (2011). Estratégias para melhorar os processos de abstração na disciplina de algoritmos. In XXXI Congresso da Sociedade Brasileira de Computação, Natal/RN. Anais do XIX Workshop sobre Educação em Computação. Natal, RN: SBC, pages 1488-1497.

Sirotheau, S., de Brito, S. R., da Silva, A. d. S., Eliasquevici, M. K., Favero, E. L., and de Lira Tavares, O. (2012). Aprendizagem de iniciantes em algoritmos e programação: foco nas competências de autoavaliação. In Brazilian Symposium on Computers in Education (Simpósio Brasileiro de Informática na Educação-SBIE), volume 1. 